\title{
El aprendizaje de la historia experimental de la química
}

\author{
José Antonio Chamizo* \\ Artículo recibido: 23-04-2009 y aprobado: 12-11-2009 \\ Learning the history of chemistry experimental
}

Un experimento más que imitar a cómo trabajan los científicos debe ser un diálogo entre el observador y el mundo natural alrededor del observador

W. De Vos

Resumen: A partir de una discusión acerca de la importancia de la experimentación en la enseñanza de las ciencias y el valor que la didáctica le da a la historia y la filosofía, se propone abordar la enseñanza experimental de la historia de la química a partir de la postura historiográfica recurrente, empleando diagramas heurísticos. Lo anterior a través de diez experimentos elegidos por su valor didáctico y tecnocientífico.

Palabras clave: Historia, química, experimentación, diagramas heurísticos y modelos.
Abstract: Based on a discussion about the importance of experimentation in science teaching and the value given to history and philosophy in Didactics, it is suggested to address the experimental teaching of Chemistry history, taking into account the recurrent historiographical view by means of heuristic diagrams. To do this, the researchers selected ten experiments due to their educational and techno scientific value.

Key words: History, chemistry, experimentation, heuristic diagrams and models.

\footnotetext{
* Facultad de Química. Universidad Nacional Autónoma de México. jchamizo@servidor.unam.mx
} 


\section{Introducción}

Ante los vertiginosos cambios que enfrentan las sociedades actuales, derivados en gran medida por el impacto que las ciencias y la tecnología tienen en la vida cotidiana de sus integrantes, se ha presentado en los últimos años, un intenso debate sobre otras formas de enfrentar los procesos de aprendizaje (Duschl, 1994; Matthews 1994; Justi, 2002). La cantidad de conocimiento científico generado es tal que no es suficiente, como se ha hecho hasta ahora, profundizar en el conocimiento específico de la asignatura correspondiente (por más que sin duda es fundamental).

Es necesario además, incorporar la reflexión sobre la estructura de las ciencias y el papel que ésta ha jugado en nuestra sociedad y, sobre todo, es necesario discutir la dinámica de cambio, puesto que lo que queremos conseguir es que los conocimientos del alumnado evolucionen hasta hacerlos rigurosos y útiles (Giere, 1988; Mc Comas, 2000). Para ello hay que escoger de entre la enorme cantidad de información generada, aquella que permita desarrollar las competencias requeridas en un mundo cada vez más cambiante y que, por ello, prepare mejor para un futuro que no está predeterminado. Aquí hay que hacer notar que ante el enorme crecimiento de la información química, que duplica todo lo conocido cada tres o cuatro años, los profesores de ciencias en general y los de química en particular (una vez que la química es la ciencia más productiva) somos, sepámoslo o no, querámoslo o no, profesores de historia de las ciencias (Chamizo, 2007a).

Este artículo integra una conferencia magistral sobre el mismo tema y un taller sobre diagramas heurísticos que se presentó en el IV Congreso Internacional sobre Formación de Profesores de Ciencias realizado en Bogotá, el pasado 2009. Aquí se abordará una nueva y diferente manera de aprender química, una manera en la que a partir del análisis del papel de la experimentación y de la historia de las ciencias en la didáctica, se reconstruye la historia experimental de la química.

\section{La experimentación}

El debate sobre los procesos de aprendizaje se ha extendido hacia el trabajo práctico en todas sus modalidades (Woolnough, 1985; Barbera, 1996; Izquierdo 1999; Psillos 2002; Chamizo, 2004), en química apela fundamentalmente a la experimentación. Dos de sus más interesantes resultados han sido, por un lado, reconocer que el propósito fundamental del mismo en la enseñanza de las ciencias, es permitir que los alumnos relacionen el complejo mundo real presente con el de los conceptos construidos a lo largo de la historia, Figura 1.

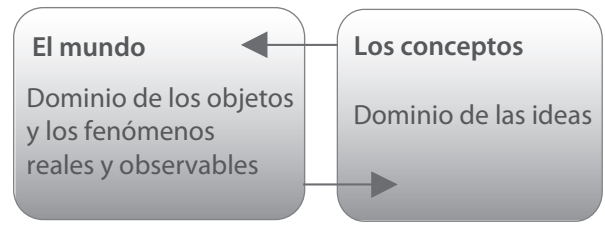

Figura 1. El propósito fundamental del trabajo práctico: permitir que los alumnos establezcan relaciones entre el mundo real y los conceptos.

El segundo resultado ha sido el replantearse la arbitraria distinción entre teoría, prácticas de laboratorio y problemas, como algo tan diferente en la enseñanza de las ciencias que dichas actividades son impartidas a menudo 
por distintos profesores (Gil, 1999). Del mismo debate sobre los procesos de aprendizaje, ha surgido un limitado acuerdo sobre los objetivos fundamentales que se persiguen al realizarlo y que aparecen como centrales en la enseñanza de las ciencias. Dichos objetivos se concretan en otras tantas actividades y son:

- Ejercicios. Diseñados para desarrollar técnicas y destrezas específicas.

- Experiencias. En las que se propone que los alumnos tomen conciencia de determinados fenómenos del mundo, sean naturales o artificiales.

- Investigaciones. En las que los estudiantes tienen que resolver un problema que, valga la redundancia, para ser problema debe ser abierto.

Hay que destacar que un mismo fenómeno puede abordarse desde cualquiera de estas actividades y por lo tanto cumplir cualquiera de sus objetivos. Para los motivos del presente artículo me centraré exclusivamente en el trabajo práctico de investigación, aquel que más se parece a la propia investigación científica y sobre el que se han tenido intensas discusiones (Kirschner, 1988;
Lewis, 2002; Caamaño, 2003; Berg, 2003; Chamizo, 2007). Aquí se reconocen las ideas pioneras de Schwab (1962) sobre los "grados de libertad" que le permitieron distinguir actividades en las que los alumnos únicamente seguían instrucciones hasta llegar a aquellas en las que ellos mismos tomaban decisiones. Herron (1971) continua en esta línea de pensamiento y es capaz de precisar los diferentes niveles que puede tener dicho trabajo (Tabla 1) en cuanto a la identificación del problema a resolver, la forma en se puede solucionar y finalmente quién lo hace.

Como se puede observar en la tabla, la inmensa mayoría del trabajo práctico que se realiza en las escuelas corresponde, cuando se lleva a cabo una investigación, a los niveles 0 y 1 . Los problemas y las formas de resolverlos son ajenos a los estudiantes, que se convierten así en meros espectadores de su potencial propio aprendizaje. Lo anterior contrasta con lo expuesto por Hodson (1994) en el sentido de que los estudiantes aprenden mejor si son activos, en lugar de pasivos.

\begin{tabular}{clll}
\hline $\begin{array}{c}\text { Nivel de } \\
\text { investigación }\end{array}$ & \multicolumn{1}{c}{ Problema } & $\begin{array}{c}\text { ¿Quién proporciona el } \\
\text { método de resolución? }\end{array}$ & Respuesta \\
\hline 0 & Profesor / a libro de texto & Profesor / a libro de texto & Profesor / a libro de texto \\
\hline 1 & Profesor / a libro de texto & Profesor / a libro de texto & Alumna/ o \\
\hline 2 & Profesor / a libro de texto & Alumno/ a & Alumna/ o \\
\hline 3 & Alumno/ a & Alumno/ a & Alumna/ o \\
\hline
\end{tabular}

Tabla 1. Marco de análisis para determinar el nivel de investigación de un trabajo práctico (Herron, 1971; Tamir, 1989 ).

\section{La historia}

La historia en general y la de las ciencias en particular, nos ha enseñando que las que fueron respuestas correctas para preguntas de su tiempo, años después, fueron consideradas erróneas. Los héroes se convirtieron en villanos y viceversa. No hay verdades absolutas: "las ciencias son más un viaje que un 
destino". En este sentido, el historiador H. Kragh (1987) reconoce al menos tres posturas en el quehacer histórico, tres diferentes estrategias historiográficas: anacrónica, diacrónica y recurrente.

En la estrategia anacrónica el pasado se estudia y se valida de manera "absoluta" a la luz del presente. Esta postura etiquetada con el nombre de interpretación "whig" ha sido ampliamente utilizada y también muy cuestionada. Por otro lado, la estrategia diacrónica consiste en estudiar la ciencia del pasado de acuerdo con las condiciones que existían realmente en ese pasado. Sin embargo el mismo Kragh indica: la historiografía diacrónica no puede ser más que un ideal. El historiador no puede liberarse de su tiempo ni evitar completamente el empleo de patrones contemporáneos.

Más recientemente, Tosh (2003) argumenta que la historia de la ciencia está inevitablemente "centrada en el presente", asunto que propuso hace años y de otra manera, el filósofo francés G. Bachelard (1972) cuando introdujo el término "historia recurrente de la ciencia" como aquella que es continuamente contada a la luz del presente. El objetivo de la historia recurrente no consiste en encontrar los conceptos que usamos actualmente en algún punto del pasado, sino en el revelar el camino por el cual esos conceptos emergieron a partir de otros conceptos en una secuencia de correcciones y rectificaciones. Cuando un nuevo concepto "aparece" introduce una reorganización de la disciplina en la cual se incorpora y una evaluación del conocimiento previo con que ésta contaba. Desde este punto de vista la ciencia se compromete periódicamente a evaluarse a sí misma, a reconocerse en su pasado. Esta historia recurrente es de- liberadamente anacrónica, pues decide si la ciencia anterior es válida, o no, a la luz de los conocimientos actuales.

La historia recurrente apela a una reconstrucción racional diferente de la propuesta por Lakatos (1978), en la cual se tiene como referencia absoluta un estándar extrahistórico de racionalidad. En este sentido es más cercana a la propuesta de Toulmin (1972, p. 95-96) y su propuesta de racionalidad moderada:

Las cuestiones de racionalidad conciernen precisamente no a las doctrinas intelectuales particulares que un hombre -o un grupo profesionaladopta en cualquier momento dado, sino a las condiciones y la manera en que está dispuesto a criticar y modificar esas doctrinas a medida que pasa el tiempo. La racionalidad de una ciencia no está encarnada en los sistemas teóricos corrientes en ella en momentos determinados, sino en sus procedimientos para llevar a cabo descubrimientos y cambios intelectuales a través del tiempo.

El mismo Kragh introduce otra forma de organización de la historia: la historia vertical y la historia horizontal. La historia horizontal aborda el cambio histórico de un concepto considerando las influencias que ha recibido y las soluciones que ha propiciado. Por otro lado, la historia vertical aborda el contexto que enmarca al concepto en un periodo de tiempo (que en caso de ser pequeño podría ser, de acuerdo con la postura del historiador francés F. Braudel, un acontecimiento). Así, un concepto específico tiene un determinado sentido cuando se presenta durante un acontecimiento particular y otro cuando el acontecimiento es diferente. Lo anterior se ilustra en la Figura 2. 


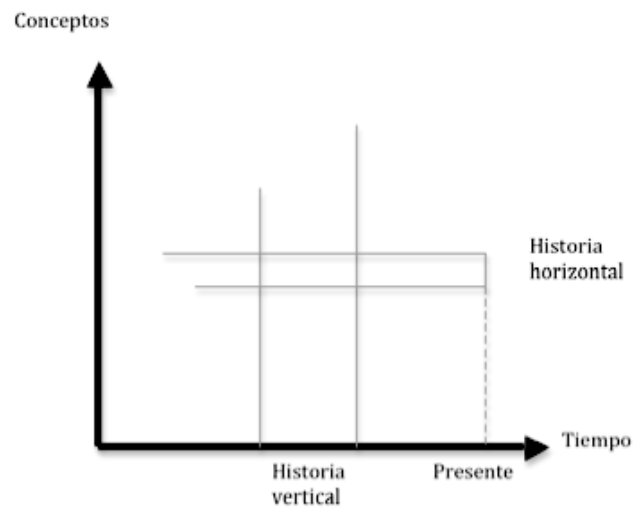

Figura 2. Maneras de organizar la historia de las ciencias: vertical y horizontal.

Con la historia horizontal se aísla un concepto asumiéndose anacrónica y recurrentemente una continuidad disciplinar. Por otro lado la historia vertical es intrínsecamente interdisciplinar y aspira a reconocer "el espíritu de la época”. Mientras que la historia horizontal constituye una película de una parte pequeña y concreta de la ciencia, la historia vertical es una fotografía de la situación general. Kragh (1987, p. 111).

Por otro lado, una revisión herética de la historia de las ciencias y la tecnología indica que ésta no se refiere únicamente a una serie de sucesiones o de remplazos de una clase de conocimiento por otra; es más una cuestión de acumulación compleja y de variedad simultánea, disputada en un cierto plazo. Compartiendo la postura del historiador de la ciencia J. Pikestone (Pickstone, 2000) en el presente estudio se reconocen tres grandes tipos de formas de conocer:

- La historia natural: que se refiere a una primera clasificación de los componentes del mundo, comprende la variedad de objetos naturales o artificiales, normales o patológicos. Es el espacio de las taxo- nomías celestes, geológicas o biológicas y del lugar donde se vuelven públicas, los jardines botánicos, los zoológicos y los grandes museos de ciencias.

- El análisis: si la variedad y el cambio son identificados por la historia natural, el análisis busca el orden por disección; aparece cuando los objetos se pueden ver como compuestos de "elementos" o cuando los procesos se pueden ver como el "flujo" de un "elemento" a través de un sistema. Es el espacio de los laboratorios de anatomía, química, física e ingeniería y de los lugares donde se vuelven públicos, las escuelas, institutos, politécnicos, hospitales y universidades.

- La síntesis: si el análisis considera el separar cosas, la síntesis trata sobre cómo ponerlas juntas. El análisis especifica la composición de lo "conocido" para posteriormente, poniendo juntos los "elementos", crear nuevos objetos o fenómenos. La experimentación se basa en la síntesis, en la producción sistemática de la novedad. Es el espacio "privado" del control, ya sea por motivos militares o económicos, de los laboratorios de biomedicina y farmacia, de diseño de nuevos materiales o de física nuclear, así como de los lugares donde se vuelven públicos, los complejos tecnocientíficos industriales.

Estas tres grandes formas de conocer, que no necesariamente son consecutivas, sirven de marco de referencia en la presentación histórica experimental de la química, es decir que diversos experimentos se identificarán en cada una, faltando por indicar la forma en que se desarrolla una determinada disciplina. La postura adoptada para este trabajo -de entre varias posibles, como la centrada en los paradigmas de Kuhn (1971)- se da a través de la resolución de problemas. El filósofo inglés S. Toulmin (Toulmin, 1977; Chamizo, 2007b) acep- 
tando el papel central de los problemas en el avance de la ciencia, avanza en la caracterización de los problemas a través de la expresión:

Problemas = ideales explicativos capacidades corrientes

Los problemas son presentados, desde esta perspectiva, en la "distancia" o "diferencia" que hay entre aquello a lo que una comunidad o un individuo aspira a comprender (ideales explicativos) y la capacidad que tiene esa comunidad o individuo para alcanzarlo, esta distancia se acorta o desaparece cuando emergen nuevos conocimientos. Ahora bien, esa aspiración se concreta generalmente a través de preguntas y las preguntas que concretan los problemas son aquellas que se refieren a su tiempo. Preguntar requiere de quien lo hace, movilizar conocimientos y habilidades y permitir el reconocimiento de la profundidad del saber. El maestro de química y filósofo de las ciencias G. Bachelard lo dijo así:

Y dígase lo que se quiera, en la vida científica los problemas no se plantean por sí mismos. Es precisamente este sentido del problema el que indica el verdadero espíritu científico. Para un espíritu científico todo conocimiento es una respuesta a una pregunta. Si no hubo pregunta, no puede haber conocimiento científico. Nada es espontáneo. Nada está dado. Todo se construye. (Bachelard, 1979, p.16)

Hace más de doscientos años, el francés A. Lavoisier no se preguntó lo mismo, por ejemplo, sobre la combustión (sus preguntas estaban centradas en la existencia e importancia del oxígeno en dicho fenómeno) que Yuan T. Lee, premio Nobel de Química en 1986, de origen chino, cuyas preguntas se centra- ban en las más de cien sustancias que se encuentran en una llama. Las preguntas y sus respuestas, los modelos -siempre tentativos- (Chamizo, 2010) y el lenguaje que se emplea para explicarlas generan conocimiento que se aplica y modifica el mundo. De esta manera, los conceptos que ahora se aprenden en las clases de ciencias son resultado de muchas preguntas, de problemas resueltos y de aplicaciones más o menos afortunadas que fueron enigmas en su momento, en pocas palabras, los conceptos son construcciones históricas.

Los conceptos científicos, cambiantes a lo largo de la historia, integran una forma tan compleja que es necesario distinguir en ellos tres características o dimensiones que, así como permitieron construirlos, van a permitir utilizarlos: el lenguaje, las técnicas de representación y los procedimientos de aplicación de la ciencia. Los dos primeros se refieren a los aspectos simbólicos de la explicación científica -esto es, la actividad científica que llamamos explicar-, es decir, una de las formas en la que hacemos públicos nuestros pensamientos, en la que una generación le transmite a otra el contenido de una ciencia, una "enculturación". Estas dos características solo tienen un uso genuinamente explicativo cuando se aplican en el mundo durante un pequeño espacio temporal, un acontecimiento. Así, la tercera característica comprende el reconocimiento de situaciones a las que son apropiadas estas actividades simbólicas, el entorno.

\section{Historia experimental de la química}

La postura historiográfica recurrente (un ejemplo de lo anterior se muestra en Chamizo 2009b) abordada en la inter- 
sección de las aproximaciones vertical y horizontal y estas tres grandes formas de conocer que, como ya se indicó, no son necesariamente consecutivas, sirven de marco de referencia para la presentación histórica experimental de la química. Sin embargo, antes de abordar el asunto es necesario destacar el reciente debate sobre los experimentos más bellos en la historia de la química.

La American Chemical Society (ACS) a través de su revista Chemical and Engeeniering News, convocó a sus lectores a escoger los experimentos más bellos en la historia de la química (Freemantle, 2003). De las 70 respuestas se identificaron 55 experimentos que recibieron al menos dos votos y solamente cinco experimentos recibieron más de tres votos. Los editores de la revista eligieron entonces a los 25 experimentos más nominados y los enviaron a un conjunto previamente seleccionado de historiadores y químicos, para que identificaran los 10 mejores. Dicha lista en orden decreciente se presenta en la Tabla 2.

Dos años después, la británica Royal Society publicó el texto de P. Ball, Elegant Solutions. Ten beautiful experiment in chemistry. Aquí el autor, conocedor de la lista publicada por la ACS, se concentra más en el sentido del experimento y la belleza privilegiando atributos como la invención, la elegancia, la perseverancia o la imaginación y cuestiona el que Lavoisier haya realizado un experimento o que Perkin se incluya en dicha lista ya que su trabajo fue fruto del azar.

Como queda claro la elección no es fácil y obedece a criterios ocasionalmente enfrentados o al menos no consensuados. Por ello es pertinente lo escrito por Sierra (2006) cuando discute sobre la reproducción de experimentos históricos:

Pero, bueno, ¿qué criterios han de considerarse a la hora de elegir un tema? En primer lugar, el valor histórico del mismo, cuya determinación se ilumina sobremanera merced al estudio y conocimiento de la historia de la ciencia y la tecnología. En segundo lugar, la posibilidad investigativa del tema, esto es, la posibilidad de proponer hipótesis, de someterlas a prueba, de construir teoría, de extraer conclusiones. En tercer lugar, la posibilidad de montaje del experimento elegido, puesto que siempre existe el riesgo en cuanto a que se hayan perdido materiales de construcción y técnicas del pasado, sobre todo las artesanales. En cuarto lugar, la posibilidad de pensar con otra cabeza... Y no se olvide lo tocante a las normas de seguridad para montar los experimentos.

El penúltimo criterio es crucial a mi modo de ver (...) o sea, la necesidad de pensar en términos de los personajes involucrados en la historia del experimento elegido. Dicho de otra manera, pensar con otra cabeza significa proceder con mentalidad de etnometodólogo sin ir más lejos, esto es, no podremos comprender un descubrimiento de, digamos, el siglo XVI si pensamos como hombres de nuestra época. 


\begin{tabular}{|c|c|c|}
\hline Freemantle (2003) & Ball (2005) & Chamizo (2010a) \\
\hline $\begin{array}{l}\text { Pasteur: separación de } \\
\text { enantiómeros (1848) }\end{array}$ & $\begin{array}{l}\text { Van Helmont: el sauce y la } \\
\text { belleza de la cuantificación }\end{array}$ & $\begin{array}{l}\text { Los metales de la antigüedad. El } \\
\text { cobre }\end{array}$ \\
\hline $\begin{array}{l}\text { Oxidación: de los metales por } \\
\text { Lavoisier (1775) }\end{array}$ & $\begin{array}{l}\text { Cavendish: el agua y la belleza } \\
\text { del detalle }\end{array}$ & $\begin{array}{l}\text { La destilación, técnica desarrollada } \\
\text { por los alquimistas árabes }\end{array}$ \\
\hline $\begin{array}{l}\text { Fisher: determinación de la } \\
\text { configuración de la glucosa } \\
\text { (1890) }\end{array}$ & $\begin{array}{l}\text { Los esposos Curie: el radio y la } \\
\text { belleza de la paciencia }\end{array}$ & $\begin{array}{l}\text { Los metales se pueden quemar. P. } \\
\text { Brun y J. Rey }\end{array}$ \\
\hline $\begin{array}{l}\text { Davy: aislamiento de metales } \\
\text { alcalinos y alcalinoterreos } \\
\text { usando electrólisis (1808) }\end{array}$ & $\begin{array}{l}\text { Rutherford: las partículas alfa y la } \\
\text { belleza de la elegancia }\end{array}$ & $\begin{array}{l}\text { La combustión para demostrar } \\
\text { que el aire es una mezcla. J. } \\
\text { Mayow }\end{array}$ \\
\hline $\begin{array}{l}\text { Perkin: colorantes de anilina } \\
\text { (1856) }\end{array}$ & $\begin{array}{l}\text { Seaborg: el seaborgio y la belleza } \\
\text { de lo pequeño }\end{array}$ & $\begin{array}{l}\text { Del aire desflogisticado de J. } \\
\text { Priestley al oxígeno de A. Lavoisier }\end{array}$ \\
\hline $\begin{array}{l}\text { Kirchoff y Bunsen: líneas } \\
\text { espectrales (1859) }\end{array}$ & $\begin{array}{l}\text { Pasteur: los cristales y la belleza } \\
\text { de la simplicidad }\end{array}$ & $\begin{array}{l}\text { La descomposición del agua por } \\
\text { electrólisis. W. Niicholson }\end{array}$ \\
\hline $\begin{array}{l}\text { Priestley: descubrimiento del } \\
\text { oxígeno (1774) }\end{array}$ & $\begin{array}{l}\text { Urey y Miller: la química } \\
\text { prebiótica y la belleza de la } \\
\text { imaginación }\end{array}$ & $\begin{array}{l}\text { La industria de los colorantes. W. } \\
\text { Perkin }\end{array}$ \\
\hline $\begin{array}{l}\text { Bartlett: Preparación de } \\
\text { compuestos de gases nobles } \\
(1962)\end{array}$ & $\begin{array}{l}\text { Bartlett: la química del xenón y la } \\
\text { belleza de lo sencillo }\end{array}$ & $\begin{array}{l}\text { El nacimiento de la } \\
\text { espectroscopia. R.W. Bunsen }\end{array}$ \\
\hline $\begin{array}{l}\text { Grignard: uso de } \\
\text { organomagnesianos en síntesis } \\
\text { orgánica (1899) }\end{array}$ & $\begin{array}{l}\text { Wodward: la vitamina B12 y la } \\
\text { belleza de la economía }\end{array}$ & $\begin{array}{l}\text { La síntesis de la Aspirina. F. } \\
\text { Hoffman }\end{array}$ \\
\hline $\begin{array}{l}\text { Los esposos Curie: } \\
\text { Descubrimiento de elementos } \\
\text { radiactivos (1898) }\end{array}$ & $\begin{array}{l}\text { Paquette: el dodecaedrano y la } \\
\text { belleza del diseño }\end{array}$ & $\begin{array}{l}\text { El nylon y las macromoléculas. } \\
\text { W.H. Carothers }\end{array}$ \\
\hline
\end{tabular}

Tabla 2. Diez experimentos en la historia de la química.

En otras palabras, la cita reconoce la importancia de la postura historiográfica recurrente (la intersección de las visiones horizontal y vertical), la posibilidad de acceso a la información histórica, los límites prácticos del mismo experimento, así como la seguridad en su realización. Por todo ello, la propuesta que se muestra en la Tabla 2 es diferente a la presentada por la American Chemical Society y la de la Royal Society. En ella se privilegia la historia en función de la didáctica (Gallego, 2007) a través de diez experimentos que permiten contar la historia de la química de manera cronológica y que pueden ser reconstruidos con aparatos modernos en un laboratorio escolar de secundaria, bachillerato o primer año de universidad. Por eso mismo algunas de las metodologías para resolver las preguntas, que dichos experimentos pueden responder, no requieren más de dos horas para llevarse a cabo. Adicionalmente, los experimentos enfrentados como investigaciones, permiten considerar la clasificación de Pickstone, es decir que pueden discutirse como historia natural, análisis o síntesis. 
Como puede observarse en la Tabla 2 , al menos tres de estos experimentos didácticos coinciden con las listas previamente publicadas; es complejo validar los otros siete de forma distinta a la mencionada anteriormente, sin embargo daré algunos indicios. El aislamiento de los metales fue fundamental en la historia de las sociedades humanas como también lo fue el desarrollo de la destilación por los alquimistas del Islam. No nos reconoceríamos sin artículos metálicos, bebidas alcohólicas, ni perfumes, pero tampoco sin saber que los metales se pueden quemar... y se queman, y que cuando esto sucede sus propiedades cambian preparando el entendimiento de la reacción química.

La lenta pero inexorable ruptura del aristotélico elemento "aire" en una confusa mezcla de gases permitió, primero aislar y luego caracterizar nuevos elementos, con lo que el análisis químico se va construyendo poco a poco. Con el advenimiento de las pilas eléctricas, la historia natural de la química se pobló, ahora si, de nuevos elementos producidos por la ruptura de diversos materiales (entre ellos el antiguo elemento agua). El azar llevó a Perkin a descubrir los colorantes artificiales y con ello, se consolidó la industria química moderna, que además contó con la contribución de la compleja pelea por la patente de la aspirina, la droga universal, ambos, acontecimientos ejemplares de la tecnociencia. Finalmente resulta imposible pensar la mecánica cuántica sin la espectroscopía, ni la modernidad material que se esbozaba en la primera mitad del siglo XX, sin los polímeros.

\section{El aprendizaje de la historia experimental de la química}

De todo lo anterior queda claro que en lugar de los comunes reportes de práctica de laboratorio y de acuerdo con lo enunciado en la Tabla 1, buscamos el nivel 2 de investigación. Para ello es posible utilizar diagramas heurísticos (Chamizo, 2009a), que no son otra cosa que "Uves de Gowin" modificadas, utilizando las propuestas filosóficas que S. Toulmin utiliza en su caracterización de los conceptos, para reconocer el aprendizaje con los trabajos prácticos de investigación (Figura 3) (Chamizo, 2007).

Los diagramas pueden ser autoevaluados según el registro de aprendizaje que se muestra en la Figura 4. Es decir, asumiendo que los diez experimentos presentados pueden abordarse como investigaciones cuya profundidad y complejidad dependerá de cada estudiante o grupo de estudiantes. Así, hay que indicar en una sola página y de acuerdo con una visión vertical de la historia (documentándolos), los hechos históricos que se conocían en ese momento y sobre los cuales se construye una pregunta (Tabla 2).

La respuesta a la misma requiere de dos acciones, por un lado, una metodología (sección derecha del diagrama) que inicia con el establecimiento de qué hacer para responder a la pregunta, llevarlo a cabo y analizar los resultados obtenidos, en este caso, a partir de la realización de un experimento; por el otro (sección izquierda del diagrama) utilizar los conceptos que se conocían en la época, es decir las aplicaciones, el lenguaje y los modelos de explicación, junto con los conceptos que se utilizan en la 
actualidad. Es importante insistir en que esta parte del diagrama heurístico es en la que se manifiesta más claramente la recurrencia, ya que se evidencian dos tiempos, el del experimento original y el del presente.

La complejidad requerida sugiere que sólo se enuncien los asuntos más significativos, siendo necesario, desde luego, comprenderlos. Mediante el uso de la metodología y de los conceptos es posible llegar a una respuesta, a un resultado; los alumnos tienen que elegir en un determinado momento de su propia investigación, cuál de estas dos opciones tomar, ya que el experimento no siempre resulta exitoso. Como se indica en el registro de aprendizaje de la Figura 4, lo anterior no disminuye la evaluación que los alumnos pueden obtener. En la Figura 5 se muestra un diagrama heurístico sobre el "descubrimiento" del hidrógeno.

\begin{tabular}{|c|c|c|c|}
\hline Diagrama heurístico sobre: & \multicolumn{2}{|c|}{ (Se refiere al tema de la investigación) } & Puntos \\
\hline \multicolumn{4}{|c|}{$\begin{array}{l}\text { Hechos } \\
\text { (Se refiere a la información obtenida y/o observaciones realizadas respecto a algo que sucede en } \\
\text { el mundo que nos lleva a formular una pregunta) }\end{array}$} \\
\hline \multicolumn{4}{|c|}{$\begin{array}{l}\text { Pregunta } \\
\text { (Enunciado de una pregunta centrada en un experimento histórico) }\end{array}$} \\
\hline Conceptos & \multicolumn{2}{|l|}{ Metodología } & 0 \\
\hline $\begin{array}{l}\text { Aplicaciones } \\
\text { (Se refiere a los usos de lo que estamos } \\
\text { investigando) }\end{array}$ & \multicolumn{2}{|c|}{$\begin{array}{l}\text { Procedimiento para la obtención de datos } \\
\text { (Se refiere a lo que hacemos para obtener la } \\
\text { información pertinente para poder contestar la } \\
\text { pregunta) }\end{array}$} & \\
\hline $\begin{array}{l}\text { Lenguaje } \\
\text { (Se refiere a los términos que } \\
\text { requerimos saber para responder la } \\
\text { pregunta) }\end{array}$ & \multicolumn{3}{|c|}{$\begin{array}{l}\text { Procesamiento de los datos para obtener un } \\
\text { resultado } \\
\text { (Se refiere al manejo de datos y resultados en tablas, } \\
\text { gráficas, diagramas etc. que resumen los datos } \\
\text { obtenidos) }\end{array}$} \\
\hline \multicolumn{2}{|c|}{$\begin{array}{l}\text { Modelo } \\
\text { (Se refiere al modelo que se usa para dar la respuesta a la pregunta, por } \\
\text { ejemplo, modelo atómico de Lewis, modelo de acidez de Arrhenius, etc.) }\end{array}$} & $\begin{array}{l}\text { Análisis y/o } \\
\text { conclusión } \\
\text { derivado de los } \\
\text { datos } \\
\text { (Se refiere } \\
\text { únicamente a } \\
\text { lo obtenido a } \\
\text { partir de los datos } \\
\text { procesados) }\end{array}$ & \\
\hline
\end{tabular}

\section{Respuesta o resultado}

(Se refiere a la explicación que responde a la pregunta, reuniendo los conceptos con la metodología o a las razones por las cuales falló el experimento, o no se puede contestar la pregunta)

\section{Referencias}

(Se refiere a libros, artículos de revistas, páginas web, etc.)

De los hechos:

De los conceptos:

De la metodología:

Autoevaluación (total de puntos)/20 puntos posibles

Figura 3. Diagrama heurístico. 
Tecné, Episteme y Didaxis No. 26, 2009

\begin{tabular}{|c|c|}
\hline Puntos & Características \\
\hline \multicolumn{2}{|l|}{ Hechos } \\
\hline 0 & No hay hechos \\
\hline 1 & Se identifican hechos \\
\hline 2 & Se identifican hechos y algunos conceptos \\
\hline 3 & Se identifican hechos, conceptos y algunos aspectos metodológicos \\
\hline \multicolumn{2}{|c|}{ Pregunta } \\
\hline 0 & No hay pregunta \\
\hline 1 & Hay una pregunta basada en los hechos \\
\hline 2 & Hay una pregunta basada en los hechos e incluye conceptos \\
\hline 3 & $\begin{array}{l}\text { Hay una pregunta basada en los hechos, que incluye conceptos y que sugiere aspectos } \\
\text { metodológicos }\end{array}$ \\
\hline \multicolumn{2}{|c|}{ Metodología } \\
\hline 0 & No hay metodología \\
\hline 1 & Hay un procedimiento que permite la recolección de datos \\
\hline 2 & Los datos son procesados, ya sea a través de tablas y/o gráficas \\
\hline 4 & Con los datos procesados se obtiene una conclusión \\
\hline \multicolumn{2}{|c|}{ Conceptos } \\
\hline 0 & No hay conceptos \\
\hline 1 & Se identifican las aplicaciones \\
\hline 2 & Se identifican las aplicaciones y el lenguaje \\
\hline 4 & Se identifican las aplicaciones, el lenguaje y el, o los modelos capaces de explicar la pregunta \\
\hline \multicolumn{2}{|c|}{ Respuesta en lugar de resultado } \\
\hline 0 & No hay respuesta \\
\hline 1 & La respuesta es muy semejante a la conclusión de la parte metodológica \\
\hline 2 & La respuesta incorpora además de la conclusión de la parte metodológica, los hechos \\
\hline 3 & $\begin{array}{l}\text { La respuesta incorpora además de la conclusión de la parte metodológica, los hechos y los } \\
\text { conceptos (particularmente el modelo) }\end{array}$ \\
\hline \multicolumn{2}{|c|}{ Resultado en lugar de respuesta } \\
\hline 0 & No hay resultado \\
\hline 1 & Se identifican los errores \\
\hline 2 & Se identifican y se explican los errores \\
\hline 3 & $\begin{array}{l}\text { Se identifican y se explican los errores, además se propone una alternativa } \\
\text { razonable de solución }\end{array}$ \\
\hline \multicolumn{2}{|c|}{ Referencias } \\
\hline 0 & No hay referencias \\
\hline 1 & Hay referencias únicamente de los hechos, o de los conceptos o de la metodología \\
\hline 2 & Hay referencias de los hechos y de los conceptos o de la metodología \\
\hline 3 & Hay referencias de los hechos, de los conceptos y de la metodología \\
\hline
\end{tabular}

Figura 4. Registro de aprendizaje para evaluar los diagramas heurísticos. 


\section{Hechos}

A mediados del siglo XVIII el escocés J: Black identificó al CO2 como "aire fijo", demostrando que un gas podía combinarse con un sólido, consolidando el derrumbe del concepto aristotélico de los cuatro elementos.

Durante el siglo XVIII se utilizó en Europa el modelo del flogisto para explicar la combustión.

En 1776 el inglés $\mathrm{H}$. Cavendish publicó los resultados de sus experimentos con un nuevo tipo de aire, el "aire inflamable", hoy conocido como hidrógeno.

\section{Pregunta \\ ¿Por qué no es posible decir que el inglés $\mathrm{H}$. Cavendish, con sus experimentos con ácidos y metales en la segunda mitad del siglo XVIII, descubrió el elemento hidrógeno?}

\begin{tabular}{lll}
\hline Conceptos & Metodología & 0 \\
\hline
\end{tabular}

\section{Procedimiento para la obtención de datos}

\section{Aplicaciones}

El hidrógeno no tenía ninguna utilidad al momento de descubrirse. Actualmente se utiliza principalmente en: el proceso Haber para la síntesis de amoníaco, la hidrogenación catalítica de aceites insaturados para producir grasas sólidas comestibles y para transformar diferentes óxidos metálicos en metales.
Se monta el equipo para realizar el experimento. En un matraz se coloca el zinc y se coloca la manguera por la que saldrá el gas en el tubo lateral. Se tapa el matraz con un tapón que tiene un embudo de separación con ácido sulfúrico. $\mathrm{El}$ otro extremo de la manguera se coloca dentro de un tubo de vidrio lleno de agua invertido en una cubeta, también con agua. Se deja caer el ácido sobre el zinc, aparecen burbujas y el agua del tubo de ensaye es desplazada por el gas producido. Se llenan dos tubos de ensaye con el "aire inflamable". Se destapa uno por uno cada tubo acercándoles un cerillo.

\section{Lenguaje}

Procesamiento de los datos para

De la época: Elemento, Flogisto. "aire inflamable"

Actual: Elemento, Hidrógeno

obtener un resultado

\begin{tabular}{|c|c|}
\hline Tubo & Intensidad de explosión \\
\hline 1 & Pequeña \\
\hline 2 & Mediana \\
\hline
\end{tabular}

Análisis y/o conclusión

\section{Modelo}

En aquella época se empleaba el modelo del flogisto para explicar la combustión, que es lo que sucedía cuando se acercaba una llama al "aire inflamable". Cavendish confundió el aire inflamable en un determinado momento con el mismo flogisto. Actualmente se reconoce al hidrógeno como el más ligero de los noventa elementos que existen naturalmente.

\section{derivado de los datos}

Se produce un gas que explota cuando se le acerca una flama. Que la segunda explosión sea más fuerte que la primera puede deberse a que en el primer tubo hay aire "común" que fue desplazado por el nuevo y diferente "aire inflamable"

\section{Respuesta o resultado}

A pesar de que se atribuye a Cavendish el descubrimiento del hidrógeno por la obtención del "aire inflamable", en realidad él nunca pensó que dicho "aire inflamable" era un elemento nuevo sino qu lo asoció más bien al flogisto, o parte del mismo. Cavendish antes y mejor que nadie describió la síntesis y las propiedades del "aire inflamable", no del hidrógeno 


\section{Referencias}

De los hechos: Leicester, H.M. (1967). Panorama histórico de la química, Alambra, Madrid.

De los conceptos: Química Nuffield. (1971). Colección de experimentos. Reverté, Barcelona.

De la metodología: Trifonov, D.N. (1980). Cómo fueron descubiertos los elementos químicos. Mir

Autoevaluación (total de puntos)/20 puntos posibles

Figura 5. Diagrama heurístico sobre el descubrimiento del hidrógeno.

\section{Conclusiones}

Una primera experiencia de esta aproximación didáctica se ha llevado a cabo en la Facultad de Química de la UNAM, de la misma, queda claro de manera preliminar, que es posible enseñar química de otra manera.

Hace casi una década Justi (2000) indicó la importancia de utilizar modelos históricos en la enseñanza de la ciencia. La presente propuesta acepta su sugerencia pero difiere de la de ella en que se soporta, para su empleo en el salón

\section{Bibliografía}

Bachelard, G. (1979). La formación del espíritu científico. México: Siglo XXI.

Bachelard, G. (1972). El compromiso racionalista. Buenos Aires: Siglo XXI.

Ball, P. (2005). Elegant Solutions. Ten beautiful experiments in chemistry. London: RSC.

Barbera, O. y Valdés, P. (1996). El trabajo práctico en la enseñanza de las ciencias: una revisión, Enseñanza de las ciencias, 14, 365-379.

Berg, C. A. R., Bergendahl, C. B., y Lundberg, B. K. S. (2003). Benefiting from an open-ended experiment? A comparison of attitudes to, and outcomes of, an expository versus an open-inquiry version of the same experiment, International Journal of Science Education, 25, 351-372. de clases, en las ideas de recurrencia de Bachelard y de desarrollo histórico de Toulmin, incorporando además como herramienta de trabajo los diagramas heurísticos.

Así, de lo aquí presentado y como lo indica el epígrafe, es posible enseñar historia, e historia de la química, propiciando el diálogo entre los alumnos y el mundo que les rodea, reflexionando y realizando algunos de sus experimentos más significativos.
Caamaño, A. (2003). Los trabajos prácticos en ciencias. En Jiménez Aleixandre (Coord.) Enseñar ciencias. Barcelona: Grao.

Chamizo, J. A. (2007a). Teaching modern chemistry through recurrent historical teaching models. Science \& Education, 16, 197-216.

Chamizo, J. A. (2007b). Las aportaciones de Toulmin a la enseñanza de las ciencias. Enseñanza de las ciencias, 25, 133-146.

Chamizo, J. A. (2009c). Los diagramas heurísticos en la enseñanza de la historia de la química. En Monroy, Z. y León-Sánchez, R. (Eds.). Epistemología, psicología y enseñanza de la ciencia. México: Facultad de Psicología, UNAM. 
Chamizo, J. A. (2009b). Modelos recurrentes. El modelo de Lewis-LangmuirSidgwick. Metl2, 103-116.

Chamizo, J. A. (2010a). Introducción a la historia experimental de la química, México, FQ-UNAM, manuscrito presentado para publicación, en http:// depa.fquim.unam.mx/SHFQ

Chamizo, J. A. (compilador) (2004). Antología de la Enseñanza Experimental. México: UNAM.

Chamizo, J. A. (2010) Una tipología de los modelos para la enseñanza de las ciencias. Revista Eureka Enseñanza y Divulgación de las Ciencias, 7, (1), 26-41.

Chamizo, J. A. e Izquierdo M. (2007). Evaluación de las competencias de pensamiento científico, Alambique, 51, 9-19.

Duschl, R. A. (1994). Research in the History and Philosophy of Science. In D.L Gabel (Ed.). New York: Handbook of Research on Science Teaching and Learning, Macmillan.

Freemantle M. (2003). Chemistry at its most beautiful. $C \&$ ENews, 25 august. 81, 34, 27-30.

Gallego, A. (2007). Ciencia, historia, epistemología y didáctica de las ciencias: las comunidades de especialistas, Tecné, Episteme y Didaxis: TED. 22, 113-125.

Giere, R. (1988) Explaining Science. A cognitive approach. Chicago: University of Chicago Press.

Gil, D., Furio, C., Valdés, P., Salinas, J., Martínez-Torregrosa, J., Guisáosla, y Pessoa, A. (1999). ¿Tiene sentido seguir distinguiendo entre aprendizaje de conceptos, resolución de problemas de lápiz y papel y realización de prácticas de laboratorio? Enseñanza de las ciencias, 17, 311-319.
Herron, D. (1971). The nature of scientific enquiry. School Science Review, 79, 171-172.

Hodson, D. (1994). Hacia un enfoque más crítico del trabajo experimental, Enseñanza de las ciencias, 12, 299-313.

Izquierdo, M., Sanmartí, N. y Espinet, M. (1999). Fundamentación y diseño de las prácticas escolares de ciencias experimentales. Enseñanza de las ciencias. 17, 45-59.

Justi, R. (2000). Teaching with Historical Models in Gilbert J.K. and Boutler C. J. Developing Models in Science Education. Dordrecht: Kluwer.

Justi, R. \& Gilbert, J. (2002). Models and Modeling in Chemical Education. In Chemical Education: Towards Research-based Practice. Dordrecht: Kluwer.

Kirshner, P. \& Meester, M. (1988). Laboratory approaches', Higher Education, $17,81-98$.

Kragh H. (1987). An Introduction to the Historiography of Science. Cambridge: Cambridge University Press.

Kuhn, T. S. (1971). La estructura de las revoluciones científicas. México: Fondo de Cultura Económica.

Lakatos, I. (1978). Mathematics, Science and Epistemology: Philosophical papers 2. Worral J. and Currie, G. (Eds.). Cambridge: Cambridge University Press.

Lewis, J. (2002). The effectiveness of Mini-Projects as a preparation for open-ended investigations. In Psillos, D. \& Niedderer. (Eds.). Teaching and learning in the science laboratory. Dordrecht: Kluwer Academic Publishers.

Matthews, M.R. (1994). Science Teaching: The Role of History and Philosophy of Science, London: Routledge. 
McComas W.F. (2000). The Nature of Science in Science Education. Rationales and Strategies, Dordrecht: Kluwer Academic Publishers.

Millar, R., Tiberghein, A. \& Le Maréchal, J.F. (2002). Varieties of Labwork: a way of profiling labwork tasks. In Psillos, D. \& Niedderer (Eds.). Teaching and learning in the science laboratory. Dordrecht: Kluwer Academic Publishers.

Pickstone, J. V. (2000). Ways of knowing, Manchester: Manchester University Press.

Psillos, D., \& Niedderer. (Eds.) (2002). Teaching and learning in the science laboratory. Dordrecht: Kluwer Academic Publishers.

Sierra, C. (2006). La reproducción de experimentos históricos en relación con la forja de ethos científico. Revista Eureka de Enseñanza y Divulgación de las Ciencias, 3 (1), 60-76.
Tamir, P. (1989). Training teachers to teach effectively in the laboratory. Science Education, 73, 59-69.

Tosh, N. (2003). Anachronism and retrospective explanation: in defence of a present-centred history of science. Studies in History and Philosophy of Science, 34, 647-659.

Toulmin, S. (1977). La comprensión humana I: el uso colectivo y la evolución de los conceptos. Madrid: Alianza Editorial.

Woolnough, B. E. \& Allsop, T. (1985). Practical work in science. Cambridge: Cambridge University Press. 